\title{
Performance Evaluation of AODV Routing Protocol in VANET with NS2
}

\author{
Ms. Divya Rathi ${ }^{1}$ Mrs. R.R.Welekar ${ }^{2}$

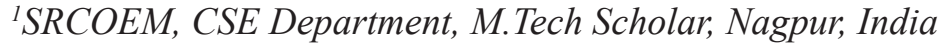 \\ ${ }^{2}$ SRCOEM, CSE Department, Assistant Professor, Nagpur, India
}

\begin{abstract}
In intelligent transportation systems, the collaboration between vehicles and the road side units is essential to bring these systems to realization. The emerging Vehicular Ad Hoc Network (VANET) is becoming more and more important as it provides intelligent transportation application, comfort, safety, entertainment for people in vehicles. In order to provide stable routes and to get good performance in VANET, there is a need of proper routing protocols must be designed. In this paper, we are working with the very well-known ad-hoc on-demand distance vector (AODV) routing protocol. The existing Routing protocol AODV-L which is based on the Link expiration time is extended to propose a more reliable AODV-AD which is based on multichannel MAC protocol. For the performance evaluation of routing protocols, a simulation tool 'NS2' has been used. Simulation results show that the proposed AODV-AD protocol can achieves better performances in forms of high Route stability, Packet Delivery ratio and packet loss rate than traditional AODV-L and traditional AODV.
\end{abstract}

Keywords - VANET, AODV, NS2, Packet Delivery Ratio, Packet Loss Ratio, Route Reliability.

\section{INTRODUCTION}

$\mathrm{T}$ HERE are various types of ad hoc networks and one of them is VANET. Vehicular Ad Hoc Network (VANET) is a subclass of Mobile Ad hoc Network (MANET). MANET and VANET have some common features such as low bandwidth and self-organization and shared radio transmission. The main work of VANET is the provision of vehicle-vehicle wireless communication and vehicle infrastructure communication (e.g., between vehicles and road side equipment), and these connections can be established without central access. The communication between vehicles has some specifics such as high speed and mobility, and that is the key feature of vehicular ad-hoc networks that makes them unique in the context of MANETs. By using vehicle to vehicle communications, drivers can be notified of important traffic data such as the condition of roads and accidents. Such information will improve drivers' decisions in hard conditions. Moreover, vehicular communications will help to monitor and manage traffic distribution and to improve vehicle fuel economy. Routing algorithms are an important part of a vehicular ad hoc network where it forward information in order to connect vehicles and having communication between them. The proposed routing protocols and mechanisms that may be employed in VANETs should adapt to the rapidly changing topology.

$\mathrm{T}_{\mathrm{N}}$ this paper, we propose a new routing scheme to make a more reliable route from source to destination node. The whole work is based on the VANET scenario, where vehicles move with different velocities along two directions on the highway. The simulation is performed to evaluate the performance of the proposed algorithm in comparison to existing AODV-L and the traditional AODV routing protocol. Packet delivery ratio and Loss packet ratio are the performance metrics considered in the evaluation process.

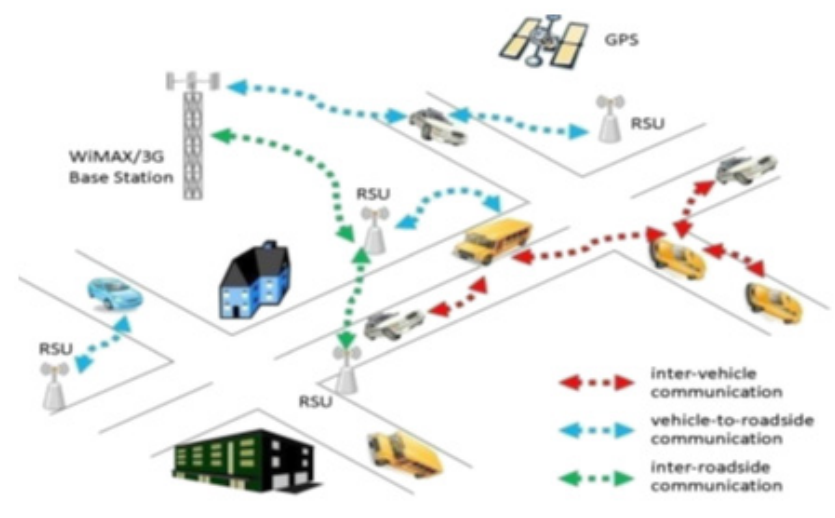

Fig1. Architecture of Vehicular Network.

\section{A. AODV routing protocol:}

AODV routing protocol is classified as a member of Bellman-Ford distant vector protocol which work in mobile network. AODV is a reactive and an on demand distance vector routing protocol, which means that that it searches a route only when there is a need of sending data packets from source to a destination. It distributes routing request packets whenever it is required due to which network overhead is very low along with that it provides loop-free routes. This protocol uses following Messages for transmission:

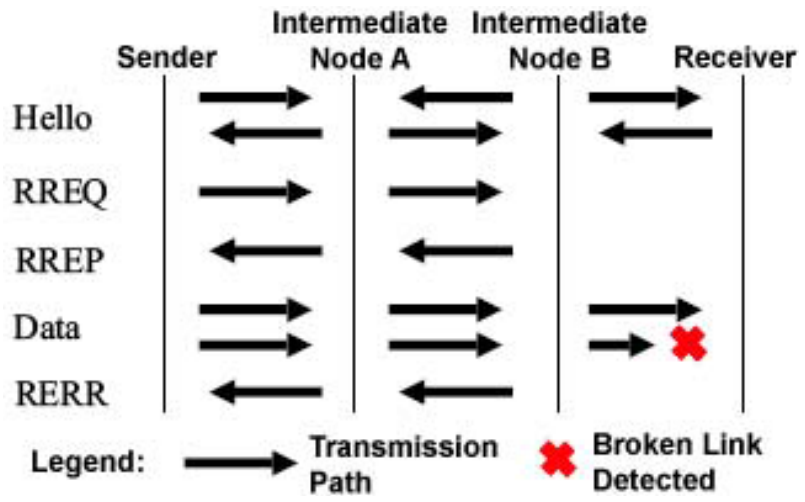

Fig 2. AODV routing message

A RREQ message is broadcasted when a source node needs to discover a route to a destination. As a RREQ propagates the intermediate nodes of the network updates their routing tables by using 
it. The RREQ also contains the most recent sequence number for the destination. When a RREQ reaches to the destination node, a route is made available by unicasting a RREP message back to the source. If it is itself the destination, then a node generates a RREP. As the RREP propagates back to the source node, middle nodes update their routing tables in the direction of the destination node direction. RERR message is broadcast for broken links. It is generated directly by a node or passed on when received from another node.

\section{Literature Survey}

In [1], author Yang He, Wenjun $\mathrm{Xu}$ and Xuehong Lin, proposes a new stable routing protocol, which is based on the scenario where vehicles move at a different velocity on the highway. The uniqueness of this work lies in its specific design that considers the vehicular motion and the channel state information between all vehicles which wants to establish links. In this way, the communication-link reliability is improved by calculating the link expiration time among the vehicles of the route from source to destination. The simulation experiments have been performed to calculate the performance of the proposed scheme in comparison to the AODV protocol. Finally, the improvement of the AODV-L is evaluated in the terms of the performance metrics packet delivery ratio and average end-to-end delay.

The survey paper [2] provides a broad overview in vehicular networking and gives a brief introduction to the limitations of the routing protocols and the challenges in designing algorithms for VANETs is to provide reliable packet transmission with minimum delay, maximum throughput, and low communication in vehicles. The authors of [3] propose a reliability-based routing system making an allowance for the mathematical distributions of movement of vehicles and the link breakages in the route. In [4], authors propose a routing protocol by make use of the vehicles movement information (e.g., position, velocity, speed, acceleration and direction) based on Ad-hoc On-Demand Distance Vector (AODV) [5] and gives its significance. In [6], the author takes on the stochastic large-scale fading model which gives results in a log-normal formula as a naive channel model.

In Hop Reservation Multiple Access [7] describes a multi-channel protocol which uses slow frequency hopping spread spectrum (FHSS) for the hosts hop from one channel to another channel in network as per to a predefined hopping configuration. First the two nodes do the handshaking by RTS/CTS and exchange the data by it, for the communication they stay in a frequency hop. Other hosts carry on hopping, and on different frequency hops more than one communication can be take place. In [8] Receiver Initiated Channel-Hopping with Dual Polling uses a same approach, but the receiver node initiates for the avoidance of the collision and do handshake process in the place of the sender. This can be done by using only one transceiver at each host in the network, but only frequency hopping networks can be applied and cannot be used in systems which use the mechanisms like direct sequence spread spectrum (DSSS). Nasipuri et al. [9] propose a multichannel CSMA protocol with "soft" channel reservation. If there are $\mathrm{N}$ channels, then according to protocol each host can listen concurrently to $\mathrm{N}$ channels. If there is an idle channel, then a host which wants to transmit packet select that channel. The preference is given to that channel which was used for the last successful transmission. In [10] the protocol is extended in order to select the preeminent channel based on signal power detected at the sender. These protocols require $\mathrm{N}$ number of transceivers for each host, which is very expensive this is drawback to this protocol.

\section{RESEARCH METHODOLOGY}

In this paper we are introducing a new system which is the enhanced part of the existing system which is based on the concept of Link Expiration Time (LET). As described further the existing system work is done in OMNET++ and we are doing work with NS2 simulator. So, whole work is divided in two parts:

1. Firstly, there is a comparison between the traditional AODV and the AODV-L routing algorithm using NS2 simulator.

2. Then making of enhanced algorithm which is the extended part of the AODV-L algorithm with the concept of multichannel MAC protocol.

\section{A. Existing System:}

In this system by using the vehicles movement information with highway mobility model based on position, direction, velocity it is predicted that how long the route is reliable [1]. There is a use stochastic large-scale fading channel model to calculate the transmission range, which should also be stochastic and the highway mobility model is used to recalculate the route lifetime. In this way, a new reliability model is proposed to facilitate the reliable routing in VANETs. The well-known ad-hoc on-demand distance vector (AODV) routing protocol is extended to propose the reliable ad-hoc on-demand distance vector routing protocol AODV-L. Simulation results is demonstrated by OMNET++ that AODVL outdoes significantly the AODV routing protocol in terms of more efficient delivery ratio and less end-to-end delay.

\section{1) Channel Model:}

\section{Stochastic Large-Scale Fading Model:}

Roadside constructions, foot-travelers and vehicles themselves may become difficulties in communication in VANET which affects the channel state among the vehicles. Moreover, due to the continuous moment of the vehicles the transmission environment also varies. Considering all these factors, there is a stochastic large scale fading channel model as follows and put the resulting distribution of these variations into the log-normal part in the formula.

$$
r x(d)=P o-10 n \log _{10} \frac{d}{d o}+N
$$

\section{Highway Mobility Model:}

The moment of the vehicles on highways depends on the high speeds of all vehicles, velocity, traffic density, the weather conditions, and the behavior of the drivers. So, by using these two models, we can achieve more accurate and reliable link between vehicles.

A macroscopic traffic flow model is a mathematical model that expresses the traffic flow characteristics like flow, density, mean speed of a traffic stream, etc. while the microscopic traffic flow models feign single vehicle-driver units, so the microscopic properties such as position and velocity of a particular vehicles is represented by dynamic variables of the models. The position of each single vehicle is needed to find that whether two vehicles come in the range of each other so that they can communicate with each other. By using the transmission power and the channel state information we can estimate the range of communication, and also the link reliability by the position and the velocity of a single vehicle.

\section{2) Route reliability definition:}

The link reliability is defined as the stable duration of the communication link between two vehicles. Link expiration time (LET) denotes the maximum time lasts from establishing the link to one vehicle moving out from the communication range. To calculate LET, speed and position of each vehicle are needed. 
Consider we have two nodes $i$ and $j$ to calculate LET of them. Let $R$ is the transmission range of every node. Distance between them is $|\mathrm{di}, \mathrm{j}|$ and velocity of each node is vi and vj. If this node moves in the same direction then, we have:

$$
L E T=\frac{R+\alpha *|d i, j|}{|v i-v j|}
$$

If two nodes are moving towards each other, means they first come closer and then go far for this condition $\alpha$ is +1 . However, if two nodes are not in the same direction means they never come closer, so there $\alpha$ is -1 . If the nodes move in the different direction, we have the following equation.

$$
L E T=\frac{R+\alpha *|d i, j|}{|v i+v j|}
$$

We can also calculate RET (Route Expiration Time) after calculating of LET. RET for a route is minimum LETs that make that route:

$$
\mathrm{RET}=\min \{\mathrm{LET} 1, \mathrm{LET} 2, \ldots \ldots . \mathrm{LETn}\}
$$

Broken link is the link which break while communication. If route has less broken link then the route is more stable. Otherwise, due to high broken link there is more exchange control packet and more packet loss.

\section{B. Proposed System:}

Here we are going to propose a medium access control (MAC) protocol for ad hoc wireless networks that utilizes multiple channels with dynamism to give better performance [18]. This multiple channels are available at the physical layer of the network. The IEEE 802.11 standard allows the use of multiple channels, but the MAC protocol is designed only for a single channel.

Designing a MAC protocol that works with the multiple channels is not an easy, as many of current devices of IEEE 802.11 have one half-duplex transceiver. This transceiver can switch the channels dynamically, but it can only communicate in one direction on single channel at a time. Thus, when a host is attending on a particular channel, it cannot take part in the communication going on a different channel. Because of this, a new type of hidden terminal problem arises in this multi-channel environment, which we can call as multi-channel hidden terminal problem. So, this single-channel MAC protocol does not work properly in a multi-channel environment where nodes may dynamically switch channels.

The scheme increases network throughput significantly, although when the network is very congested. By using multiple channels, we can get a higher network throughput than using one channel, because by multiple channels there is a multiple transmission can be done without interruption of other. So, we are going to propose a MAC protocol which can enable the hosts to dynamically change the channels such that multiple communications can be done in the same region at the same time, in different channel. As we are going to work with an ad hoc network that does not depend on infrastructure, so there is no central ability to perform management of different channels. In our work the main idea is that, we have to divide the time into fixed-time intervals by using beacons, and also having a small window at the starting position of each interval to get information about the traffic and accordingly change channels for use during the interval.

To improve the throughput there are other several MAC protocols are also proposed. But due to multiple channels they require multiple transceivers per host or they not able to overcome from the multi-channel hidden terminal problem, which does not give good performance. This is the protocol that needs only one transceiver per host, but still it can solve the hidden terminal problem in a multi-channel environment. As the protocol requires one transceiver per host, we are able to implement

\begin{tabular}{|c|c|c|}
\hline $\begin{array}{l}\text { Sir } \\
\text { no. }\end{array}$ & Existing System & Proposed System \\
\hline 1. & $\begin{array}{l}\text { This system works with the LET } \\
\text { concept to find the reliable route. }\end{array}$ & $\begin{array}{l}\text { This system is the extended } \\
\text { work of the existing system with } \\
\text { multichannel concept. }\end{array}$ \\
\hline 2. & $\begin{array}{c}\text { Single channel is present between } \\
\text { nodes. }\end{array}$ & $\begin{array}{l}\text { Multiple channels are assigning } \\
\text { between nodes. }\end{array}$ \\
\hline 3. & $\begin{array}{l}\text { Routes selected based on metrics } \\
\text { like hop distance, signal strength, } \\
\text { degree of stability and expected } \\
\text { transmission time. }\end{array}$ & $\begin{array}{l}\text { Load balancing between } \\
\text { available channels is done }\end{array}$ \\
\hline 4. & There is no such guarantee. & $\begin{array}{l}\text { Guarantees route establishment } \\
\text { if the route can be established in } \\
\text { a single channel network with } \\
\text { same topology. }\end{array}$ \\
\hline 5 & $\begin{array}{c}\text { It gives moderate result as } \\
\text { compare to new proposed } \\
\text { system. }\end{array}$ & $\begin{array}{l}\text { As having multiple channels its } \\
\text { throughput will increase and give } \\
\text { more efficient result with respect } \\
\text { to our performance metrics. }\end{array}$ \\
\hline
\end{tabular}
it with a hardware complexity which is comparable to IEEE 802.11.

TABLE 1: COMPARISON TABLE FOR BOTH THE SYSTEMS:

IV. Simulation Setup and Results:

In this section first we are considering the performance metrics considering which the enhancement is done. After it the description of the simulation environment and the first part of the expected outcome is presented. It has the results of the comparison between the traditional AODV routing protocol and the AODV-L routing protocol by using simulator NS2.34.

\section{A. Performance Metrics:}

Packet Delivery Ratio: Packet delivery ratio is a very important factor to measure the performance of protocol in any network. Packet delivery ratio is the ratio of number of packets received at the destination node to the number of packets sent from the source node. The performance is better when packet delivery ratio is high. Mathematically it can be shown as equation.

Loss Packet Ratio: Packet Loss Ratio is the ratio of the number of packets that never reached the destination to the number of packets originated by the source. Mathematically it can be shown as equation.

Route Stability: The link reliability is defined as the stable duration of the link between two vehicles.

\section{B. Simulation Environment:}

Here we are comparing the above mentioned three performance metrics. Our simulation has been done in a $1000 \mathrm{~m} \times 1000 \mathrm{~m}$ area. Vehicles are randomly placed on the road and go straightly ahead in high velocity until they reach intersection. The static nodes which are the roadside unit do not move. The simulation experiment is conducted on NS2.34 and IEEE 802.11. The transmission rate is of $2 \mathrm{Mbps}$ and transmission range s of $200 \mathrm{~m}$ used as MAC protocol. The number of vehicles considered is $30-50$. Here the source node is the UDP agent and Destination node is Null agent communicates using 512 CBR (Constant Bit Rate). 
TABLE I. COMPARED PERFORMACE METRICS

\begin{tabular}{|c|c|}
\hline Parameter & Value \\
\hline Network Simulator & NS2.34 \\
\hline Simulation Area & $1000 \times 1000 \mathrm{~m}$ \\
\hline CBR & $512 \mathrm{bytes} / \mathrm{sec}$ \\
\hline 802.11 rate & $2 \mathrm{Mbps}$ \\
\hline Transmission Range & $200 \mathrm{~m}$ \\
\hline No. of vehicles & $30-50$ \\
\hline No. of roadside units & $8-10$ \\
\hline Simulation time & $500-1000 \mathrm{sec}$ \\
\hline
\end{tabular}

Based on the simulation result we have generated the graph which shows the performance differences between AODV and AODV-L. The graphs are given below. These graphs are generated for 30 nodes but at the different velocity $(20,40,60,80,100 \mathrm{~ms})$. Figure 3 is of the Packet Delivery Ratio at different velocities shows that the pdr of AODV-L is more than AODV. Figure 4 is of the packet loss ratio shows that plr of AODV-L is less than AODV. Figure 5 is for the route reliability of these protocols of which AODV-L give more efficient result than AODV routing protocol.

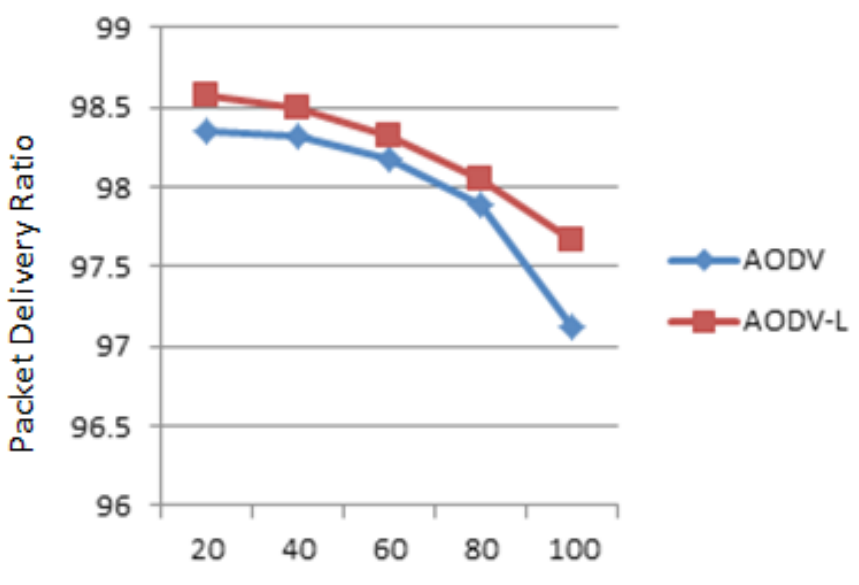

Speed $(\mathrm{m} / \mathrm{s})$

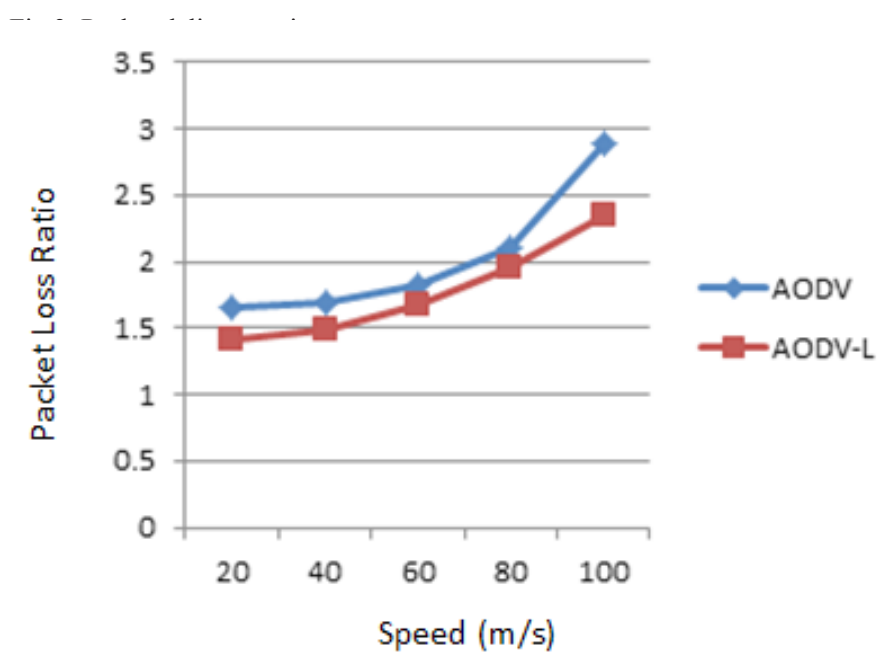

Fig 4. Packet loss ratio.

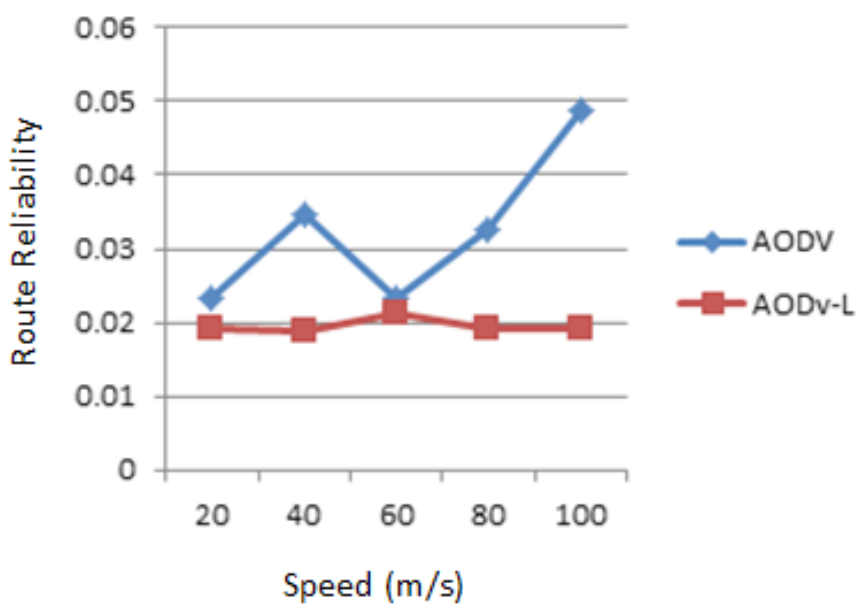

Fig 5. Route reliability.

\section{Conclusion}

In this paper, firstly, we introduce about the existing system which that enhances the stability and reliability of the routing protocol in VANETs. The idea behind the offered scheme AODV-L is the Highway Mobility Model and the Stochastic Large- Scale Fading Model with the applied concept of calculation of LET, which is the strategy for the selection of stable routes. This protocol gives the effective Simulation results of this part of the work in terms of packet delivery ratio, loss packet ratio and route reliability. In the future work, we will carry out more intensive simulation by adding the concept of multichannel MAC protocol to this AODV-L routing protocol. This scheme will give more effective output in terms of throughput and also the considered parameters.

\section{ACKNOWLEDGMENT}

I would like to express my gratitude towards my mentor Ms. Rashmi Welekar, Assistant Professor in Department of Computer Science and Engineering from Ramdeobaba College of engineering and management, Nagpur for her guidance and encouragement which helped me to understand such a vast topic of Vehicular Ad-hoc Networks. I would like to thank my family as well, for their continuous support and encouragement.

\section{REFERENCES}

[1] Yang $\mathrm{He}$, Wenjun $\mathrm{Xu}$ and Xuehong Lin, "A stable routing protocol for highway mobility over vehicular ad-hoc networks", IEEE 2015.

[2] G. Karagiannis, O. Altintas, E. Ekici, et al., "Vehicular networking: A survey and tutorial on requirements, architectures, challenges, standards and solutions," IEEE Commun. Surveys \& Tutorials, vol. 13, no. 4, pp. 584-616, 2011.

[3] M. H. Eiza, Q. Ni, T. Owens, et al.,"Investigation of routing reliability of vehicular ad hoc networks," EURASIP journal on wireless communications and networking, vol. 1, pp. 1-15, 2013.

[4] H. Guo, F. Tao, M. Ma, et al., "A reliable route selecting algorithm for vehicle communication," 2009 IEEE 7th International Conference on Information, Communications and Signal Processing (ICICS), 2009, PP. $1-5$.

[5] S. R. Das, E. M. Belding-Royer, and C. E. Perkins, "Ad hoc on-demand distance vector(AODV) routing," 2003.

[6] N. Akhtar, O. Ozkasap, and S. C. Ergen, "VANET topology characteristics under realistic mobility and channel models," 2013 IEEE Wireless Communications and Networking Conference (WCNC), 2013, pp. 1774 1779 . 
[7] Z. Tang and J. J. Garcia-Luna-Aceves, "Hop-Reservation Multiple Access (HRMA) for Ad-Hoc Networks," in Proc. of IEEE INFOCOM, 1999.

[8] A. Tzamaloukas and J.J. Garcia-Luna-Aceves, "A Receiver-Initiated Collision-Avoidance Protocol for Multi-Channel Networks," in Proc. of IEEE INFOCOM, 2001.

[9] A. Nasipuri, J. Zhuang and S. R. Das, "A Multichannel CSMA MAC Protocol for Multihop Wireless Networks," in Proc. of IEEE Wireless Communications and Networking Conference (WCNC), September 1999.

[10] A. Nasipuri and S. R. Das, "Multichannel CSMA with Signal Power-based Channel Selection for Multihop Wireless Networks," september2010.

[11] N. Akhtar, O. Ozkasap, and S. C. Ergen, "VANET topology characteristics under realistic mobility and channel models," 2013 IEEE Wireless Communications and Networking Conference (WCNC), 2013, pp. 17741779.

[12] V. Namboodiri and L. Gao, "Prediction-based routing for vehicular ad hoc networks," IEEE Trans. Veh. Tech., vol. 56, no. 4, pp. 2332-2345, 2007.

[13] L. Cheng, B. E. Henty, D. D. Stancil, et al., "Mobile vehicle-to-vehicle narrow-band channel measurement and characterization of the $5.9 \mathrm{GHz}$ dedicated short range communication (DSRC) frequency band," IEEE $J$. Sel. Areas Commun., vol. 25, no. 8, pp. 1501-1516, 2007.

[14] O. Renaudin, V. Kolmonen, P. Vainikainen, et al., "Non-stationary narrowband MIMO inter-vehicle channel characterization in the 5-GHz band," IEEE Trans. Veh. Tech., vol. 59, no. 4, pp. 2007-2015, 2010.

[15] N. Jain and S. Das, "A Multichannel CSMA MAC Protocol with ReceiverBased Channel Selection for Multihop Wireless Networks," in Proc. of the 9th Int. Conf. on Computer Communications and Networks (IC3N), October 2001.

[16] F. A. Tobagi and L. Kleinrock, "Packet Switching in Radio Channels: Part II - the hidden terminal problem in carrier sense multiple-access modes and the busy tone solution," IEEE Transactions on Communications, COM-23, 1975.

[17] A. Nasipuri, S. Ye, J. You and R. Hiromoto, "A MAC Protocol for Mobile Ad Hoc Networks using Directional Antennas," in Proc. of IEEE Wireless Communications and Networking Conference (WCNC), Chicago, IL, September 2000.

[18] J. So and Nitin Vaidya," Multi-Channel MAC for Ad Hoc Networks: Handling Multi-Channel Hidden Terminals Using A Single Transceiver", May 2004

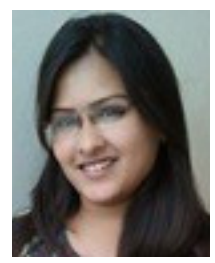

Divya Rathi she is a student of Master of Technology in Computer science engineering at Ramdeobaba college of Engineering and Management, Nagpur. She has received her Bachelor of Technology degree in Computer Science from Sant Gadagebaba University, Amravati. Her area of interest is networking and she published one survey paper in International conference named RICE 2016.

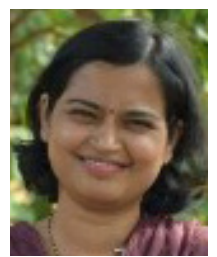

Rashmi R. Welekar, she is working as Assistant Professor at Ramdeobaba college of Engineering and Management, Nagpur. She has been teaching from last 10 years in the field of Computer Science \& engineering. She did his M. Tech from Nagpur University. Her areas of interest are Image Processing, Networking, and Pattern Recognition. She has about 10 research papers published in International conference. Journals, 6 international conferences and 1 national 